\title{
EI boletín ASCUBI Villa Clara, reflejo del acontecer informativo en el centro de Cuba
}

\section{The Bulletin ASCUBI Villa Clara, a Reflection of the Informative Events in the Central Part of Cuba}

\author{
Maylin Frías Guzmán \\ Universidad Central "Marta Abreu" de Las Villas, Cuba. \\ Luis Ernesto Paz Enrique \\ Universidad Central "Marta Abreu" de Las Villas, Cuba. \\ Cristina Alomá Cires \\ Biblioteca Provincial "Marti" de Villa Clara, Cuba. \\ Deymis Tamayo Rueda. \\ Universidad Central "Marta Abreu" de Las Villas, Cuba. \\ Eduardo Alejandro Hernández Alfonso. \\ Universidad Central "Marta Abreu" de Las Villas, Cuba.
}

Recibido: 30 de octubre de $2017 \quad$ Aceptado: 23 de febrero de 2018

Publicado: 08 de febrero de 2019

Resumen: los boletines de las asociaciones bibliotecarias en Cuba alcanzan gran auge a partir de la década de 1930. ASCUBI Villa Clara surge en 1990 como una publicación desarrollada en el ámbito de una provincia en la región central de Cuba. El boletín intentó recoger algunos detalles históricos de la asociación. Sobre ASCUBI Villa Clara solo se encuentran anotaciones descriptivas sobre la composición, estructura y personalidades vinculadas a su publicación. Se plantea como objetivos valorar la contribución del boletín ASCUBI Villa Clara para la profesión bibliotecaria en el territorio y analizar la estructura de la publicación desde lo técnico, lo formal y lo comunicativo. Se identificaron varios aspectos históricos de la publicación a partir del análisis de los documentos y la entrevista a las personas involucradas con la publicación. Además, se especificaron aspectos técnicos, formales y comunicativos que caracterizaron el boletín. 
Palabras Clave: boletín ASCUBI Villa Clara, Filial ASCUBI Villa Clara, análisis histórico, análisis comunicativo, boletines de asociaciones profesionales de bibliotecarios.

\begin{abstract}
Cuba reach great height from the decade of 1930 of the past century. ASCUBI Villa Clara arises in 1990 as a publication developed in the area of a province in the central region of Cuba. The bulletin tried to collect some historical details of the association. About ASCUBI Villa Clara, there are only descriptive annotations about the composition, structure and personalities linked to its publication. The objectives are to evaluate the contribution of the ASCUBI Villa Clara bulletin for the library profession in the territory and to analyze the structure of the publication from the technical, formal and communicative aspects. Several historical aspects of the publication were identified from the analysis of the documents and the interview with the people involved with the publication. Were specified technical, formal and communicative aspects that characterized the bulletin?
\end{abstract}

Keywords: ASCUBI Villa Clara bulletin, ASCUBI Villa Clara association, historical analysis, communicative analysis, professional library associations bulletins.

\title{
I. Introducción
}

Las publicaciones seriadas tienen la función de socializar información de carácter general y de tipo científico técnico. Por sus contenidos y formatos de presentación, las publicaciones pueden clasificarse en informativo-culturales o seriadas y científicas (Figura 1). Los boletines divulgativos son una herramienta de comunicación para la difusión de novedades, utilizada por organizaciones, empresas y profesionales para promocionar sus productos y servicios. Son considerados indispensables para promocionar el acontecer de las organizaciones e instituciones, con el objetivo de mantener una secuencia de entrega a clientes o usuarios y colaboradores que, de alguna manera, están vinculados con la actividad que estos realizan.

El uso de boletines divulgativos se ha generalizado en las asociaciones profesionales como complemento para socializar actividades, cursos y eventos de las especialidades a las que tributan. Las secciones más frecuentes en los boletines son las noticias y las convocatorias. El uso de estas fuentes de información resulta oportuno por su rápida difusión. Por lo general, los contenidos que se socializan en boletines no son evaluados por pares. La frecuencia puede variar, pero usualmente circulan semanal y mensualmente, a diferencia de las revistas. 
Figura 1

\section{Clasificación de las publicaciones seriadas}

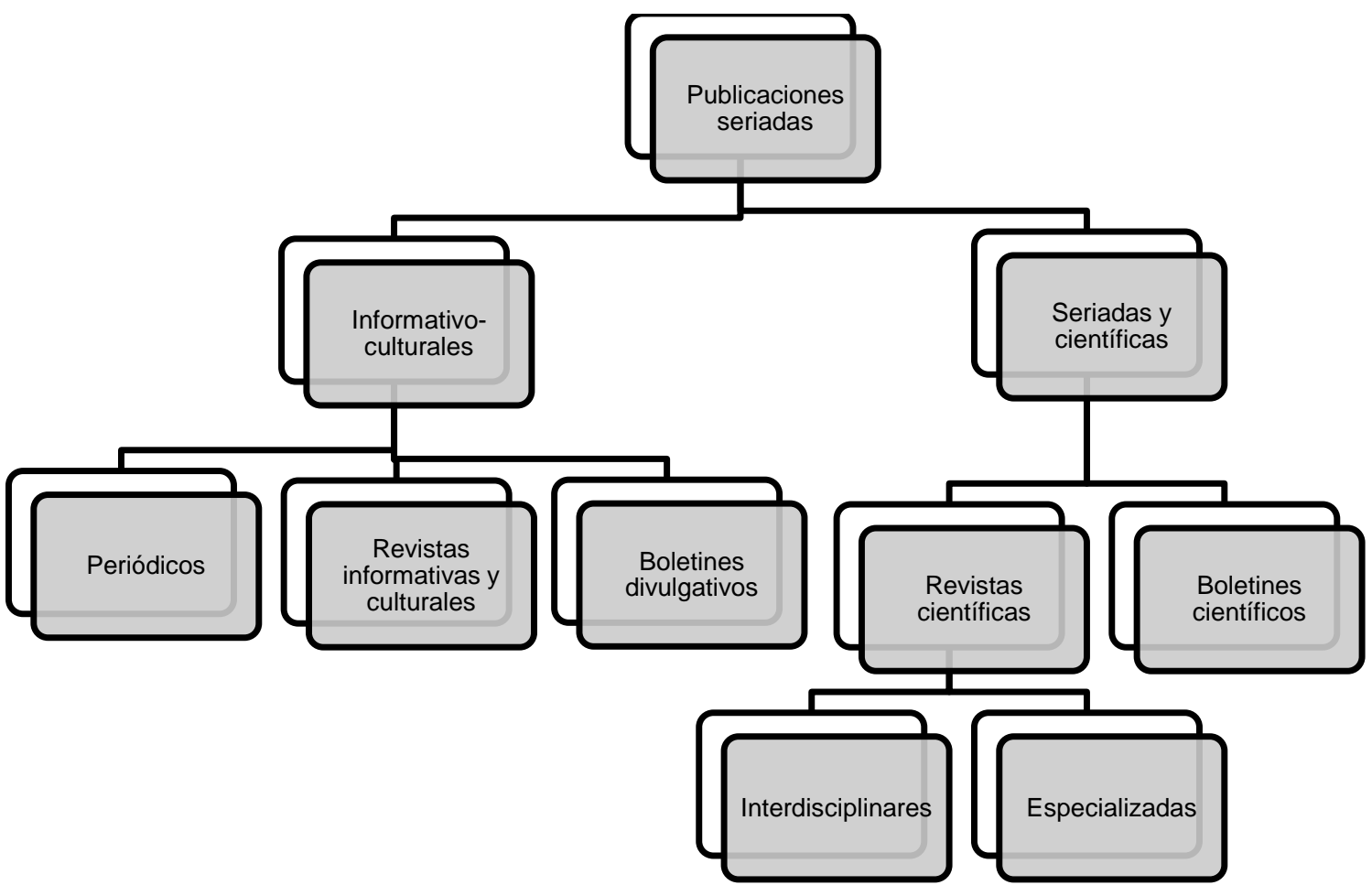

Fuente: elaboración propia de los autores (2018).

Existe un uso recurrente de boletines divulgativos en asociaciones de profesionales bibliotecarios y de información en sentido general. Una de las publicaciones de la tipología mencionada, más reconocidos en habla hispana, es el Boletín de la Asociación Andaluza de Bibliotecarios. En América Latina se destaca el Boletín de la Asociación Argentina de la Información (Boletín SAI) editado por el Instituto de Investigaciones Bibliotecológicas.

El análisis de boletines permite develar los aspectos principales contenidos y las menciones de responsabilidad de estos. Por un lado, los elementos formales de la comunicación favorecen la identificación de las principales secciones, colaboradores y temáticas. Por otro lado, el análisis del diseño editorial debe caracterizar la correspondencia con los atributos, convivencia, tipografía, interlineado, logotipo, ícono, colores empleados y figura asociada a la identidad de la publicación. 


\section{Antecedentes y problema de investigación}

La creación y proliferación de órganos de propaganda, en el marco de las asociaciones de bibliotecarios en Cuba, constituyó un fenómeno usual. Su objetivo era divulgar el quehacer científico y cultural y debatir sobre las temáticas vigentes de la profesión. La génesis de los órganos de propaganda fue la década de los 30 del siglo XX y en el transcurso del tiempo, entre las principales publicaciones seriadas que florecieron se encuentra el Boletín Bibliotécnico como resultado de la Asamblea Pro-Bibliotecas.

Otra publicación fue la Revista Cuba Bibliotecológica de la Asociación Nacional de Profesionales de Biblioteca (ANPB) y el Boletín de la Asociación Cubana de Bibliotecarios (ACB), el Anuario Bibliográfico Cubano, del cual Pérez (2005) expone que fue la primera publicación bibliológico-informativa en Cuba. La publicación respondió al movimiento bibliográfico que se desató en el país a finales del siglo XIX y que marcó cierto predominio en los primeros 30 años del siglo XX en la literatura informativa.

Las referencias a los órganos de propaganda y divulgación de las asociaciones de bibliotecarios cubanas se encuentran fusionadas en las investigaciones históricas relativas a las asociaciones profesionales del sector bibliológico-informativo de Hernández (2002) y Bellas (2011). Se han establecido alusiones al tema en el estudio del fenómeno bibliotecario cubano desde diferentes aristas como las de Frías (2008), Rivera (2008) y Viciedo (2005). También constituye un referente esencial la investigación de Vivero, Rivera, Linares y Botana (2007) sobre la revista Cuba Bibliotecológica en el intento de determinar su aporte al desarrollo de la Bibliotecología cubana en la década del 50 del siglo XX. El estudio describió en una base de datos la totalidad de los artículos, reseñas de libros y noticias presentes y derivó un análisis bibliométrico y análisis de contenido que reveló las tendencias del fenómeno bibliotecario cubano en el contexto de la época. Se destacan otros estudios como los de Bellas (2006), Bellas (2007), Montes de Oca y Rivera (2006), Paz (2015), Pedroso (2005), Pérez (2005), Pérez (2007) y Rivera (2005).

Con el triunfo revolucionario se fundó en 1963 el boletín Bibliotecas que posteriormente asume el formato de revista científica bajo el nombre de Bibliotecas. Anales de Investigación. La fundación de la Asociación Cubana de Bibliotecarios (ASCUBI) fue en 1986. En 1989 se crea el boletín ASCUBI Informa, importante exponente de la tradición en 
la divulgación bibliotecaria. Su versión electrónica se oficializó en el 2003, ajustada a las tendencias de la edición y publicación contemporáneas. La investigadora Bellas (2008) plantea que "El Boletín ASCUBI Informa pretende ser algo imprescindible para nuestros asociados, nos encontramos trabajando para que cada día en sus líneas aparezcan cuestiones de interés para todos los bibliotecarios y trabajadores de la información" (p. 2).

En el año 1990 se da a conocer el órgano de divulgación oficial de la filial con forma de boletín, titulado ASCUBI Villa Clara. La filial villaclareña fue una de las pocas en contar con un boletín oficial. El boletín ASCUBI Villa Clara es uno de los documentos en el ámbito provincial que intentó recoger algunos detalles históricos de la asociación. El boletín fue el homólogo del boletín nacional ASCUBI Informa. Vale destacar que de lo que se conoce, esta fue la única filial con una publicación de tipo informativo para sus asociados. Sobre el boletín ASCUBI Villa Clara solo se encontraron anotaciones descriptivas en la investigación de Aparicio (2012) sobre la composición, estructura y personalidades vinculadas a su publicación. La intención de ampliar el alcance del estudio derivó en la presente propuesta que pretende valorar la contribución del boletín ASCUBI Villa Clara para la profesión bibliotecaria en el territorio y analizar la estructura de la publicación desde lo técnico, lo formal y lo comunicativo.

El estudio del boletín como publicación distintiva de una organización profesional ofrece una visión de las prácticas editoriales manifiestas en la estructura y elementos estéticos que lo componen. Revela el pensamiento teórico y la actividad práctica de los profesionales del campo bibliotecario en el territorio en un espacio temporal. Deriva en una contribución al rescate de la identidad, las tradiciones y valores del conglomerado de individuos que representa y favorece la memoria histórica y al patrimonio cultural de la provincia de Villa Clara.

\section{Metodología}

El estudio corresponde a una investigación histórica teniendo en cuenta que aborda acontecimientos del pasado vinculados a la creación y evolución de una publicación. Se ajustó a una perspectiva cualitativa dominante o principal (Hernández, Fernández y Baptista, 2006) en el que se incorporaron elementos cuantitativos. El boletín ASCUBI Villa Clara resultó la principal unidad de análisis, constituida por tres números solamente. La 
necesidad de ahondar en el fenómeno hizo que se seleccionara una muestra no probabilística de casos- tipo, formada por asociados que estuvieron vinculados a la edición de la publicación o coincidieron en el mismo espacio- tiempo en el que se sucedieron los hechos.

En la investigación se emplearon los siguientes métodos del nivel teórico y empírico.

- Inductivo-deductivo: asistió al análisis e interpretación de los datos obtenidos, lo que permitió arribar a inferencias relativas al fenómeno que se estudia, especialmente en los boletines de asociaciones profesionales.

- Analítico-sintético: se realizó una revisión de la bibliografía obtenida. Se sintetizaron aspectos relacionados con los boletines de asociaciones profesionales y su presencia en la provincia de Villa Clara.

- Histórico-lógico: permitió fundamentar los resultados a partir del contexto histórico y contextual.

- Sistémico-estructural: permitió la estructuración lógica de los contenidos, se jerarquizaron criterios teniendo en cuenta la generalidad de los conceptos y se organizó la información presentada partiendo de lo general a lo particular.

- Análisis documental clásico: se consultaron para la fundamentación del tema en cuestión, diferentes fuentes de información, fundamentalmente las ediciones de los boletines que circularon en la Filial ASCUBI Villa Clara.

- Análisis de contenido: permitió la caracterización del boletín atendiendo a los siguientes niveles:

1. Alcance: temática y periodicidad que caracterizaron los números del boletín. Subcategorías: 1) función, 2) objetivos y 3) frecuencia.

2. Personalidades: sujetos responsables 0 vinculados a la elaboración del boletín. Subcategorías: 1) miembros del Comité Editorial y 2) autores de los artículos y secciones.

- Análisis de redes sociales: permitió determinar las relaciones sociales que emergieron de la interacción entre los miembros del comité editorial del boletín. Favoreció la distinción de grupos e individuos que constituyeron entes representativos en el gremio al que correspondía la publicación.

- Método bibliométrico: permitió la caracterización de la producción científica del boletín, en correspondencia con su corto período de existencia. A partir de este 
método se sistematizaron aspectos históricos que se corroboraron con entrevistas realizadas a firmantes y miembros del Consejo Editorial del boletín. Las variables e indicadores empleados se muestran en la Tabla 1.

\section{Tabla 1}

Variables e indicadores empleados para el boletín ASCIBI Villa Clara

\begin{tabular}{|l|l|}
\hline Variable & Indicadores \\
\hline Autor & Número de producciones por autor \\
\hline Temática & Mapa cognitivo de palabras en el título \\
\hline
\end{tabular}

Fuente: elaboración propia de los autores.

- Entrevista a profundidad: permitió intercambiar en varias sesiones con informantes claves para ahondar sobre la sucesión de los hechos que conforman la memoria histórica de la Asociación. Se elaboró una guía semiestructurada de asuntos o preguntas que permitió introducir los temas y ofrecer flexibilidad para indagar hasta donde se consideró necesario y pertinente.

- Método estadístico-matemático: se apoyó en la presentación de los resultados mediante expresiones porcentuales, gráficos y elementos de la Estadística Descriptiva. Se elaboraron matrices de co-ocurrencia de personalidades para la representación de sus relaciones vinculadas al fenómeno que se estudia.

Se aplicaron diversas técnicas que permitieron recoger información como la revisión bibliográfica, entrevista y la triangulación de información. Se emplearon los softwares Excel 2007, Ucinet y NextDraw para el procesamiento de los datos, lo cual permitió la representación y visualización de los resultados. Se empleó para el análisis del diseño editorial del boletín ASCUBI Villa Clara, los procederes metodológicos declarados en Hernández y Paz (2016), específicamente los indicadores relacionados con los elementos visuales:

- Marca verbal: denominación, nombre que refiere a lo semántico.

- Marca visual: se compone de tipografía, logotipo, ícono, figura asociada a la identidad de la publicación. 
- Correspondencia con los atributos: refiere al concepto central del diseño, para lo cual se deben integrar las herramientas y recursos para transmitir el mensaje.

- Convivencia: refiere a la coherencia de los elementos empleados en el diseño del producto con el objetivo para el que fue diseñado.

- Análisis formal: constituye una descripción sintética de los principales elementos que componen el diseño: tipografía, interlineado, ícono, colores empleados.

- Comportamiento en blanco y negro: refiere a la variación cromática que se les asigna a los diseños de identidad corporativa para su utilización en positivo o negativo. Contempla desde la escala de grises hasta el empleo del banco y negro como elemento distintivo.

- Pregnancia-Síntesis: integración armónica de los elementos del diseño para que estos sean correctamente decodificados por los usuarios.

\section{Resultados y discusión}

\section{a) Panorámica del boletín ASCUBI Villa Clara}

ASCUBI Villa Clara constituyó el boletín que fungió como órgano de la filial de ASCUBI en Villa Clara. Su primer número se produjo en el mes de junio de 1990 en saludo al Día del Bibliotecario. Solo se logró la publicación de tres números, que en el caso de los dos últimos se fusionaron en una misma edición y abarcaron los años de 1991 y 1992.

En la declaración de sus objetivos fundamentales se hizo notorio el alcance desde el punto de vista institucional, profesional y geográfico: “...brindar información, divulgación y promoción de las tareas llevadas a cabo en bibliotecas, centros de información y documentación y archivos..." (Aguiar, 1990, p. 2). Además, se propuso: “...dar a conocer aspectos de los que acontece en Villa Clara en la actividad científico- informativa y a la vez mantener vivo el espíritu y la comunicación entre todos los que de una forma u otra contribuyen al desarrollo y fortalecimiento del trabajo relacionado con la información científica en el territorio central" (boletín ASCUBI Villa Clara, 1992, p. 2)

No se circunscribió al acontecer de un solo tipo de institución de información, práctica informativa y área geográfica. Concibió la actualización de los sistemas de información de todo el territorio villaclareño en cuanto a sucesos actuales e históricos y aplicaciones 
prácticas. Atendió el aspecto ético y estético en función de motivar el fomento de una identidad local en los profesionales de la provincia. El Boletín se mecanografió en ambas ediciones. Su formato fue de $160 \times 208 \mathrm{~mm}$, en la primera edición, de 215 X $285 \mathrm{~mm}$ en la segunda. La paginación fluctuó entre las 20 y 25 páginas. La tipografía era de 10 puntos y esencialmente se emplearon caracteres en correspondencia con el teclado de máquinas de escribir utilizadas.

El diseño de la cubierta se caracterizó por sobriedad y sencillez. Mostró una foto en blanco y negro del Conjunto Escultórico "Ernesto Ché Guevara". Este monumento es símbolo de Santa Clara y constituye un elemento identificativo del territorio. A decir de Aguiar (1991): "Los directivos de la Asociación Cubana de Bibliotecarios, filial Villa Clara acordaron que sería la figura del "Ché" la más digna presentación de nuestro Boletín Informativo y de ahí que su portada presente el Conjunto Escultórico Ernesto Ché Guevara" (p. 3). La Figura 2 muestra la portada del boletín de la filial.

La ausencia de gráficos e imágenes estuvo condicionada por la escasez de recursos para su elaboración. Se intentó mantener una estructura homogénea mediante secciones permanentes como: Editorial o Nota de Redacción, Actividades de la ASCUBI y Noticias. Dedicó un apartado a caracterizar alguna institución de información del territorio o su actividad en particular. Fue característico el intercalar notas informativas o frases entre los diferentes apartados.

En el encabezado se reproduce en letras mayúsculas las iniciales de la Asociación y el nombre de la ciudad. Según Aguiar (1991), las siglas representan “... con justeza a todos los dedicados a la actividad de información que un día en la provincia decidieron voluntariamente unirse a trabajar por el desarrollo de esta actividad y fortalecer el trabajo relacionado con la información científica" (p. 3). El esfuerzo de la Dirección Provincial del sectorial de Cultura en Villa Clara, a través de la figura del Licenciado Andrés Chávez Molina permitió la salida de esta publicación. A este se sumó el trabajo colectivo de importantes personalidades del mundo bibliotecario e informativo de entonces. 
Figura 2

\section{Portada del boletín ASCUBI Villa Clara}

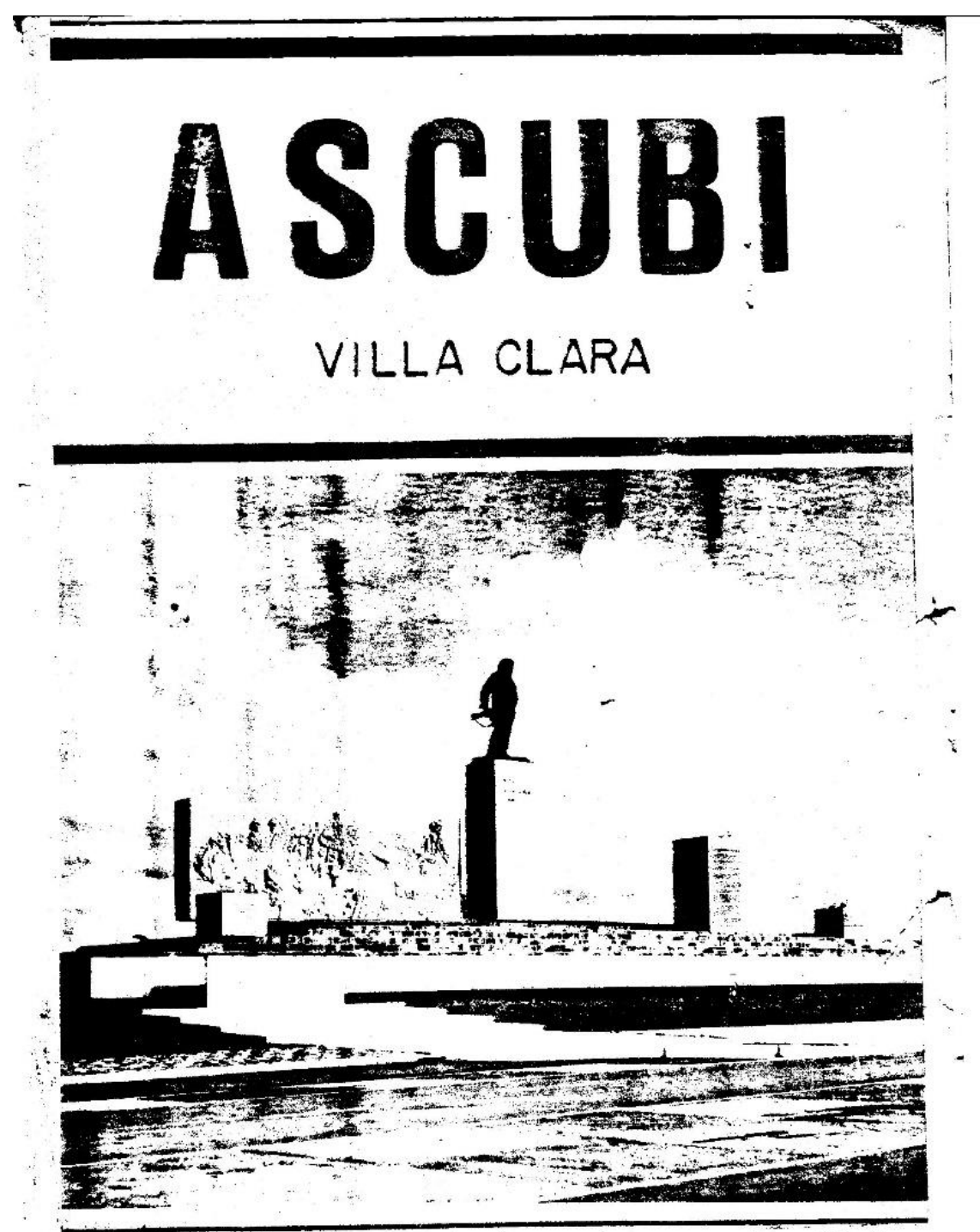

Fuente: Boletín ASCUBI Villa Clara (1992).

La Figura 3 muestra las relaciones entre los miembros del Consejo de Redacción a partir de su participación en uno u otro número. Los nodos más grandes, en el centro, representan a los profesionales responsables de ambas ediciones, destacándose con líneas gruesas el vínculo estrecho entre ellos a partir de su contribución reiterada. La variación de los miembros se ilustra en dos grupos, los nodos pequeños de la izquierda corresponden con los que se incorporaron en el primer número, los de la derecha, al segundo. 
Figura 3

Relaciones entre los miembros del Consejo de Redacción del boletín ASCUBI Villa Clara

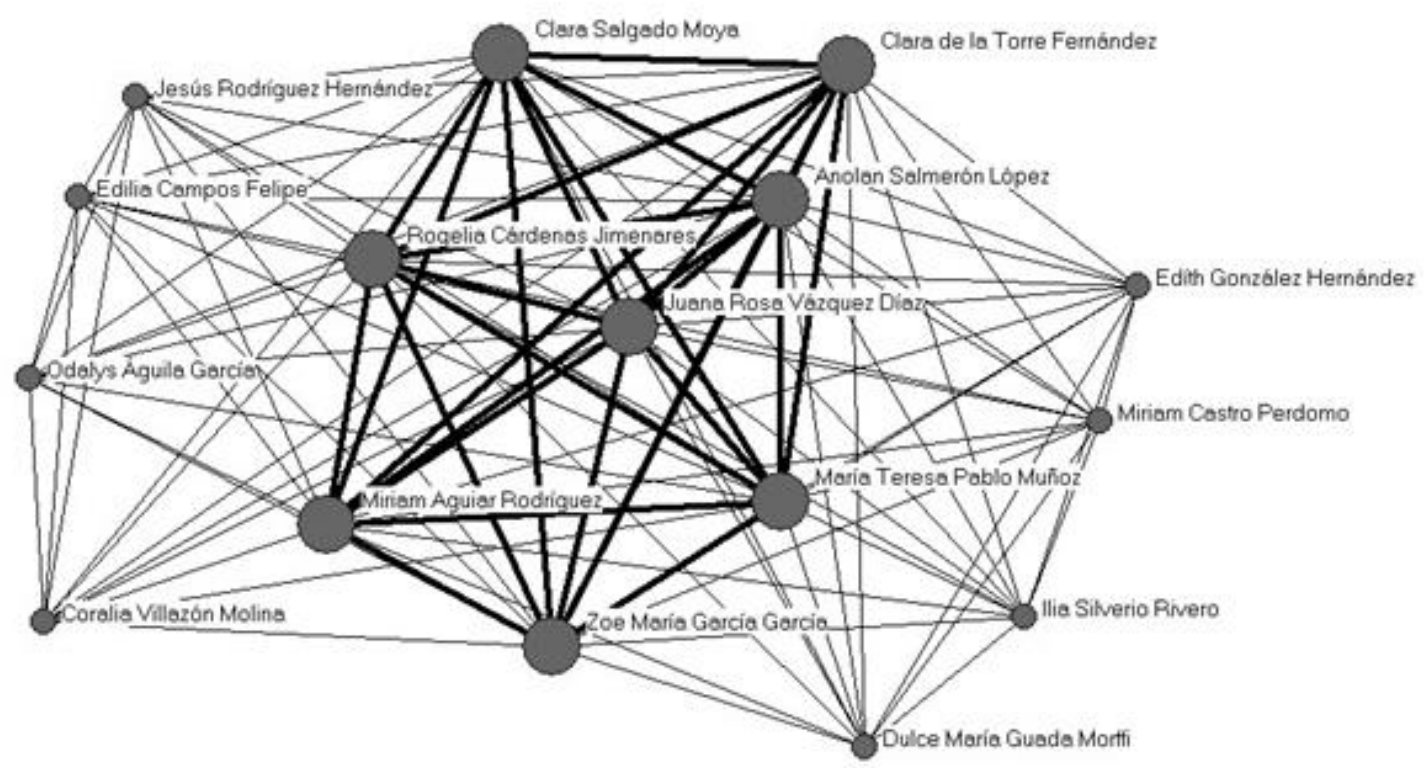

Fuente: elaboración propia de los autores.

La dirección y subdirección estuvo a cargo de Juana Rosa Vázquez Díaz y Anolan Salmerón López, respectivamente. María Teresa Pablo Muñoz, Miriam Aguiar Rodríguez, Clara Salgado Moya, Clara de la Torre Fernández y Rogelia Cárdenas Jimenares se mantuvieron como miembros en ambas ediciones. Edilia Campos Felipe (Responsable de Publicación) y Coralia Villazón Molina solo formaron parte del colectivo del primer número, mientras que llia Silverio Rivero se incorporó en el segundo. La producción gráfica fue responsabilidad de Zoe María García. En la primera edición la acompañó Jesús Rodríguez Hernández y como mecanógrafa Odalys Águila García. En la segunda Miriam Castro Perdomo, Edith González Hernández y Dulce María Guada Morffi.

De manera general, existió una ausencia de firmas en las contribuciones que formaron parte de las secciones. Se evidenció la endogamia editorial a partir del predominio de representantes del Consejo de Redacción como autores. La Figura 4 muestra la distribución. 


\section{Figura 4}

\section{Firmas de las contribuciones del boletín ASCUBI Villa Clara}

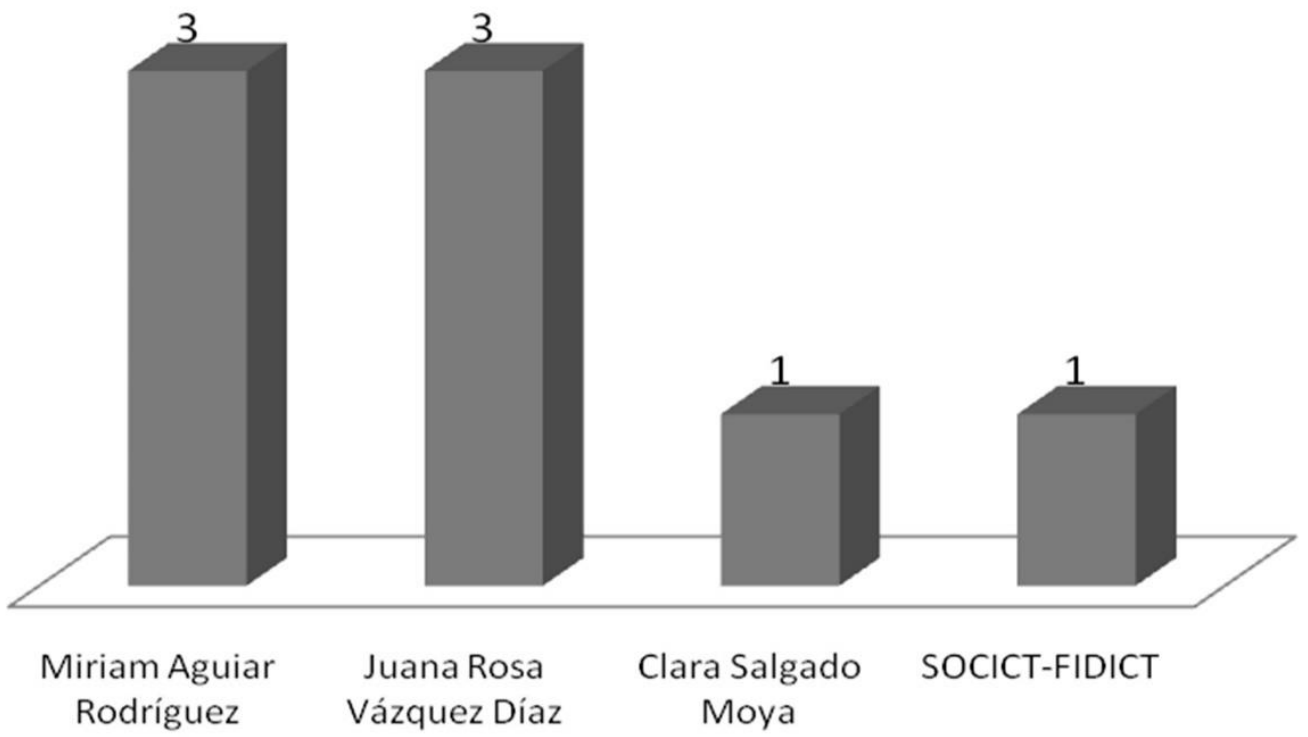

Fuente: Elaboración propia de los autores.

Miriam Aguiar Rodríguez, con tres firmas, estuvo a cargo de la Editorial que presentó el boletín y de la sección de actividades de la ASCUBI en el primer número. En el segundo expuso los argumentos para la selección del Conjunto Escultórico Comandante Ernesto Che Guevara como portada del boletín, sus características arquitectónicas, su significado, autor y ejecutor de dicha obra. Aguiar fue una de las principales promotoras del boletín desde sus funciones como primera presidenta de la Filial ASCUBI Villa Clara y asesora y coordinadora provincial de bibliotecas públicas del Ministerio de Cultura (MINCULT).

Juana Rosa Vázquez Díaz, con tres firmas, fue directora del boletín y una de las fundadoras del Buró Ejecutivo Provincial de la ASCUBI en 1987. Fue reconocida por su intensa labor divulgadora que se inicia en la Asociación y se asume de manera voluntaria en la Biblioteca Provincial "Marti". Sus contribuciones corresponden a citas, noticias y un "Breve acercamiento a Antonio Bachiller y Morales en el 7 de junio: Día de la Biblioteca", en el primer número. Sus méritos como profesora de generaciones de bibliotecarios, investigadora y bibliotecaria fundamentaron la propuesta del premio que lleva su nombre y se otorga la Filial de la ASCUBI en Villa Clara a instituciones y personalidades destacadas en el ámbito cultural e informacional del país y del territorio. 
Clara Salgado Moya fue miembro del Consejo de Redacción del boletín. "La Bibliografía" constituyó su contribución, en correspondencia con sus responsabilidades en el Departamento de selección y adquisición de la Biblioteca Provincial "Marti" y el grupo provincial de selección de la Empresa del Libro. Otra de las personas que contribuyó por el mantenimiento de la publicación fue Clara de la Torre, quien también figuraba en el comité editorial.

La Sociedad Cubana de Ciencias de la Información (SOCIT) y la Filial del Instituto de Documentación e Información Científico-Técnica y Tecnológica de Villa Clara (FIDICT) firman un artículo que reseña desde el punto de vista histórico el surgimiento del polo científico en la provincia. Este apartado constituye una de las primeras referencias, en el contexto local, sobre los productos y servicios de información especializados propuestos a la suplir las necesidades informativas de las instituciones de investigación.

Una de las secciones permanentes en todos los números fue la mención de la directiva de la filial ASCUBI en Villa Clara. La sección servía para que los afiliados conocieran su directiva. En el número uno se menciona en consejo fundador de la filial y a las personas que ocuparon los cargos en el momento en que se elaboró el boletín (Figura 5).

\section{Figura 5}

\section{Consejo provincial fundador y actual de la ASCUBI}

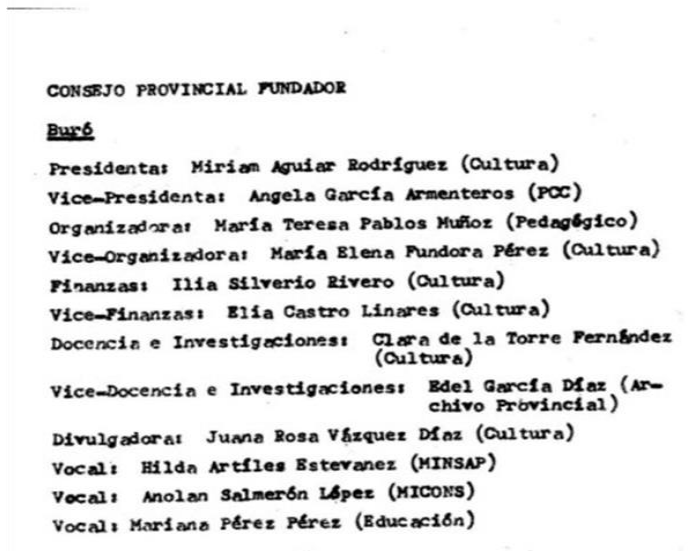

Fuente: Boletín ASCUBI Villa Clara (1990).

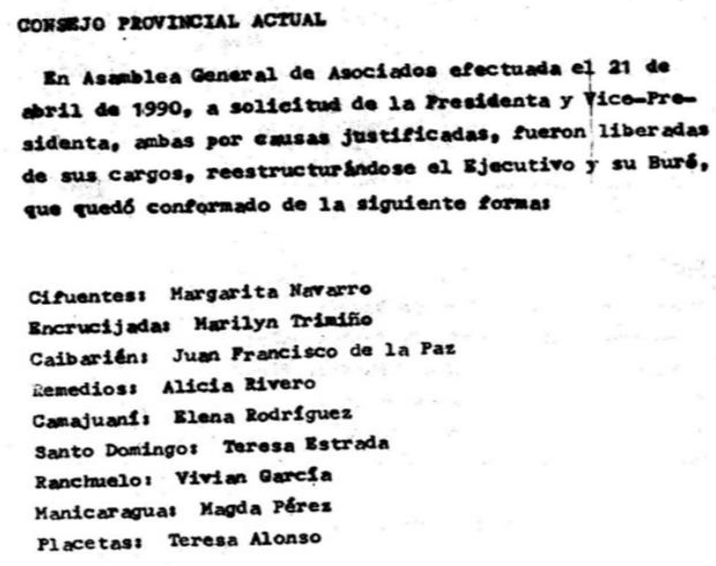

COHSEJO PRONINCTAL ACTUAL

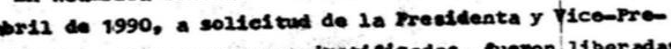


En ambas ediciones, se intentó lograr un alcance temático heterogéneo y representativo del quehacer informativo-documental del territorio. La Figura 6 ilustra las más representativas a partir de la relación de palabras clave en el título de las secciones y contribuciones del boletín.

"Bibliografía" constituyó el término más destacado a partir de la preocupación por referenciar literatura profesional y villaclareña para la actualización de los usuarios a los que se dirigió la publicación. Las siglas "ASCUBI" sobresalen en correspondencia con la propiedad intelectual del Boletín y la representación del protagonismo de los asociados en los espacios informativo-documentales del territorio. "Comité" alude a la estructura de sectores institucionales en los que se organizaba la actividad informativa de entonces. El carácter informativo y divulgativo condicionó la aparición de "actividades" y "noticias" del acontecer nacional y provincial, siendo estos, dos términos destacados. Las "bibliotecas" públicas constituyeron asunto de algunas sesiones como sistemas de información tradicionales y de mayor presencia en la región. Sobresale como núcleo temático la Información Científico Técnica ("ICT"), expresión de la atención que comienza a dársele a su organización y difusión vinculada a los centros de información y la actividad empresarial.

Aunque no resulta muy representativo en los resultados obtenidos, es importante destacar que en el boletín se divulgaban cursos de superación para los profesionales de la información en la provincia. Los cursos eran organizados por la ASCUBI. A criterio de Aguiar (2016) el proyecto principal de la Asociación era la superación de los trabajadores de la Información Científico Técnica y la Bibliotecología que era como se le llamaba, y su primer proyecto era unir a todos los especialistas, técnicos y obreros y personas afines de la actividad en la superación constante. Se ofertaron cursos de Idioma inglés, Edición de documentos, Promoción de la lectura y Metodología de la investigación. Fue significativo el postgrado sobre la Bibliografía Cubana Contemporánea a cargo del Licenciado Tomás Fernández Robaina, especialista del departamento de Investigación de la Biblioteca Nacional José Martí. 
Figura 6

Nube de palabras para la distribución temática del boletín actividades

B ib li g g r a fi a Curso disciplinario documentarios Editorial Educacion Efemerides Ernesto Escultorico Estetica Experiencia forma Fundador Guevara informativo junio Morales Noticias

Servicio Sistema Superior Tecnico tecnicos XXX

Fuente: elaboración propia de los autores.

Sobre los cursos ofertados, Alomá (2016) expresa que se realizaron cursos sobre promoción de la lectura, de comunicación social, sobre idioma inglés, sobre técnicas comunicativas, sobre desarrollo de colecciones, sobre técnicas periodísticas, sobre computación se dieron muchísimos y sobre bibliometría. Un ejemplo de los cursos ofertados aparece en el primer número del boletín ASCUBI Villa Clara (Figura 7).

El boletín ASCUBI Villa Clara constituyó un ejemplo del intento vehemente por elevar el prestigio y reconocimiento de la profesión, en una década caracterizada por la crisis económica. La dedicación y el esfuerzo de las personalidades involucradas se vieron frustrados ante la escasez de recursos materiales y la publicación dejó de existir. La creación de un boletín para la filial representó el cumplimiento de una de las mayores aspiraciones, significó poder unir todas las delegaciones de base en una publicación y divulgar el acontecer informativo villaclareño. El boletín contribuiría al desarrollo de la 
profesión en la provincia y a la divulgación de actividades del sector, pero lamentablemente desaparece en el año 1992.

\section{Figura 7}

\section{Ejemplo de curso socializado en el boletín}

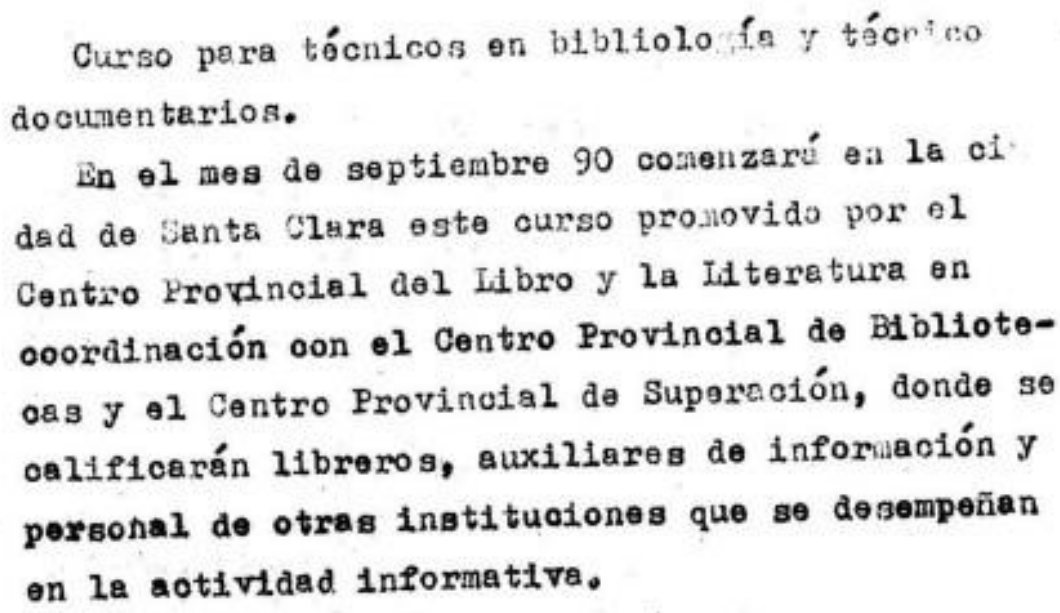

Fuente: Boletín ASCUBI Villa Clara (1990).

A partir del surgimiento de este boletín, aparecen otros medios de divulgación electrónicos para la filial. En el año 2011 y como parte de las actividades de la XI Jornada Bibliotecológica provincial de Villa Clara se presenta el estudio "Boletín Informativo: una herramienta indispensable de comunicación de la Biblioteca Provincial Marti" (Figura 8) de Rosario Cárdenas Pérez. A decir de la autora: "el boletín es una poderosa herramienta de marketing que nos da la oportunidad de promocionar todos los productos y servicios que se ofertan" (Cárdenas, Estévez y Hernández, 2011, p. 2). 


\section{Figura 8}

\section{Identificador del Boletín Informativo}

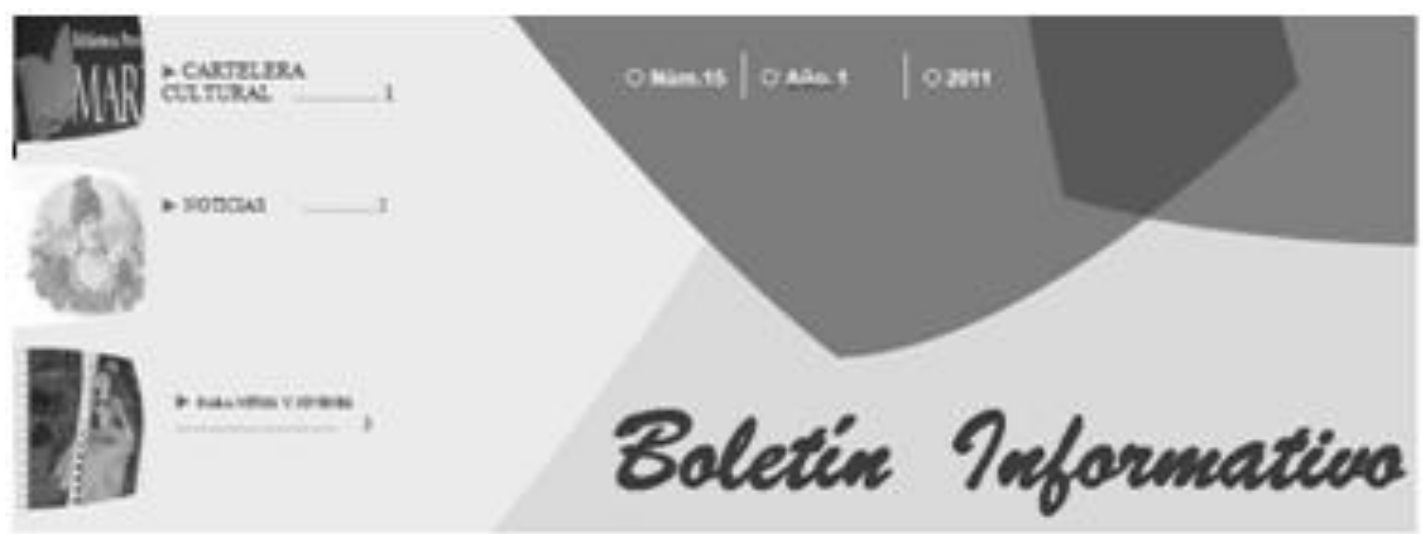

Fuente: Cárdenas, Estévez y Hernández (2011).

El boletín contaba con varias secciones que variaban en dependencia de las actividades programadas, cursos, eventos y noticias. Existían otras secciones dedicadas a promover los valores de los fondos raros y libros de diversos autores que constituyen el fondo de préstamo externo. El boletín circuló solo por tres años, dando paso al boletín Exlibri de la Sala de Fondos Raros y Valiosos de la Biblioteca Provincial "Marti" de Villa Clara. En el año 2015, a propuesta de la directiva de la filial en la provincia, se realiza un trabajo de diploma de pregrado en la especialidad de Ciencias de la Información para diseñar un boletín científico informativo para la filial (Figura 9).

\section{Figura 9}

\section{Identificador del boletín Reportes de Información}

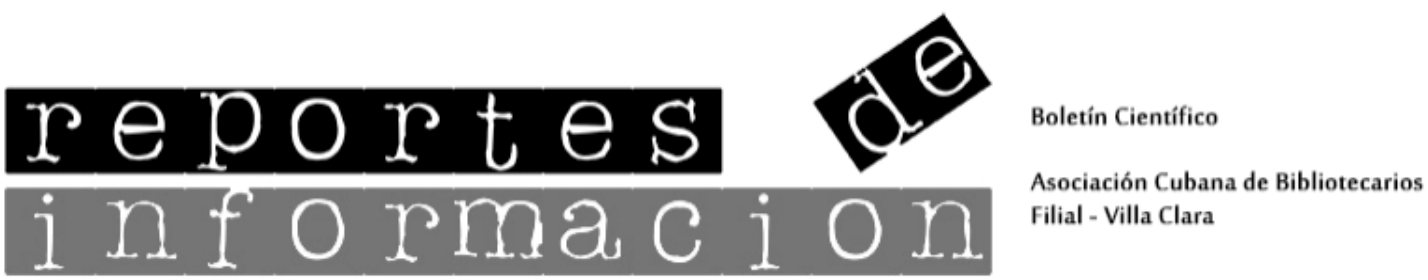

Fuente: Paz, Hernández, Tamayo y Frías (2016).

El boletín logra implementarse, pero actualmente solo cuenta con visibilidad desde la Universidad Central "Marta Abreu” de Las Villas, Cuba. 


\section{b) Análisis del diseño editorial del boletín ASCUBI Villa Clara}

Atendiendo a la marca verbal del boletín se identifica que la denominación ASCUBI, inherente a las siglas de la Asociación Cubana de Bibliotecarios, representa de manera oficial a todos sus integrantes. El efecto de representación en los profesionales de la asociación está estrechamente vinculado al proceso de asignación y connotación de la marca verbal. En la marca visual del boletín se observa en la portada de los tres números que circularon en el período 1990-1992 y aparecen inmutables dos elementos que distinguieron la publicación. En la región superior y centrada con tipografía sans serif bold la palabra ASCUBI y en la región inferior, delimitado por una línea negra y al ancho de la página la imagen del Conjunto Escultórico "Ernesto Ché Guevara".

La tipografía y el diseño presentaron variaciones en los números que circularon. Ejemplo de los cambios en los índices entre el número uno y el dos-tres se observan en la Figura 10.

\section{Figura 10}

\section{Diferencias en cuanto al diseño editorial reflejado en los índices}

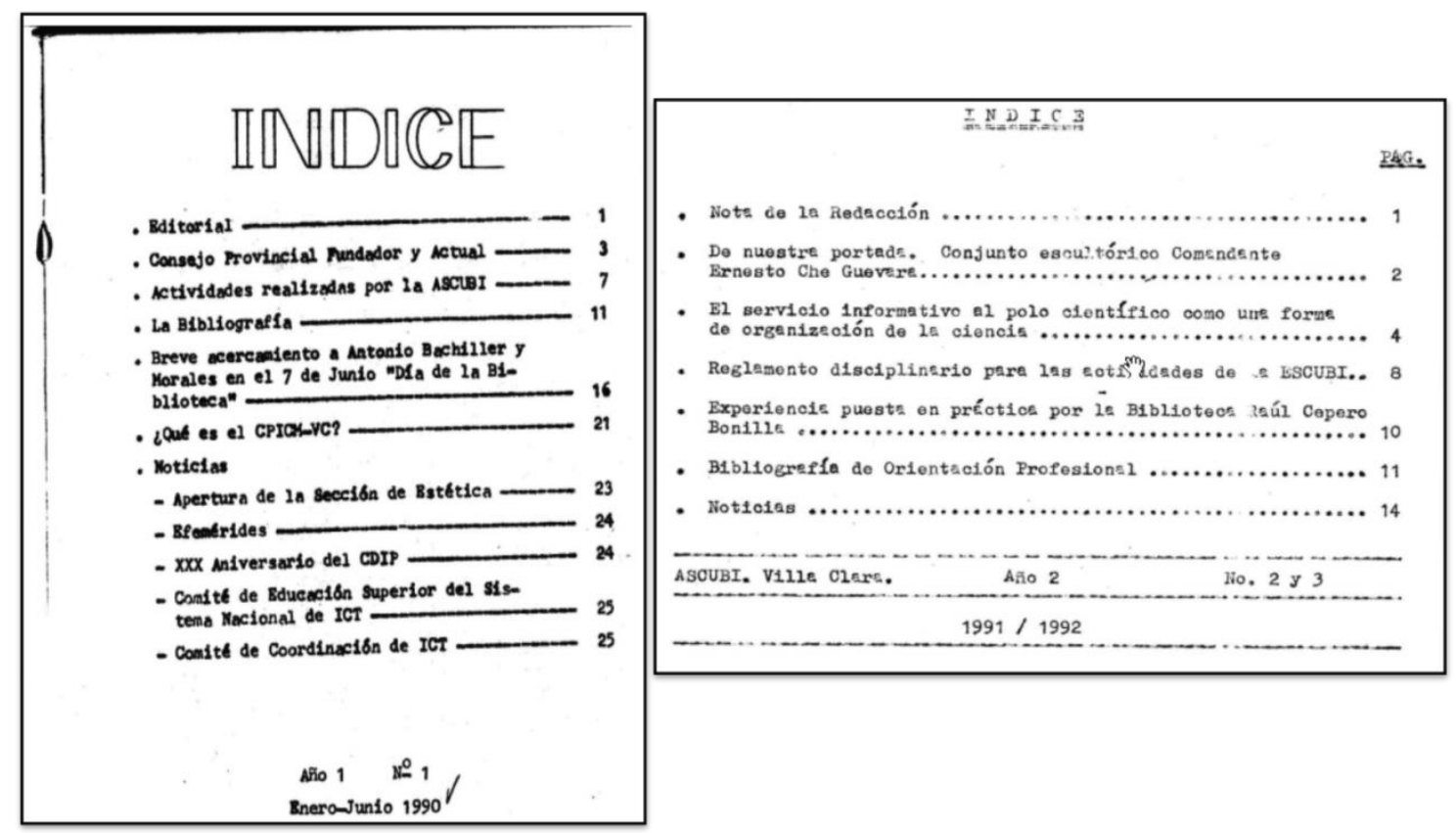

Fuente: Boletín ASCUBI Villa Clara (1990) y Boletín ASCUBI Villa Clara (1992).

A decir de varios colaboradores de la publicación seriada, hubo ausencia de una investigación (estudios de usuarios) realizada en el período 1990-1992; con la finalidad de 
instituir un imagotipo estándar para la publicación. Esta ausencia pudo haber influido en los bajos niveles de participación de las distintas delegaciones de base.

En cuanto a la selección y distribución de los elementos gráficos en la concepción del diseño, se observa un facilismo premeditado. Desde el punto de vista iconográfico e identitario, oriundo de la provincia, no resulta la figura de Ernesto Guevara lo que distingue a la filial. La connotación de la figura del Che, reviste gran importancia para todos los cubanos. Aunque la imposición de su iconografía ya sea mediante la fotografía de Alberto Korda o de su posterior monumento en la ciudad de Santa Clara, como imagen distintiva de diversas instituciones, no favorece la búsqueda objetiva de la identidad de la filial.

Atendiendo a la correspondencia con los atributos la marca formalmente no contiene ningún rasgo de conceptualización. Se identifica que existe disonancia con la imagen que le sucede en el diseño de portada. La selección de las siglas ASCUBI y el conjunto escultórico no guardan relación. No se complementan, no se declara el contenido semántico de la abreviatura, lo que dificulta el proceso de decodificación de los usuarios, teniendo en cuenta que podía ser consultado por otro profesional no perteneciente al sector.

Desde la Convivencia se establece que la marca de la Asociación Cubana de Bibliotecarios, instaurada en el año 1986, no se tuvo en cuenta en la confección de la publicación villaclareña. Lo anterior se expresa en la ausencia de un estudio de productos similares como lo fueron el boletín ASCUBI Informa del año 1989, antecedente directo del boletín ASCUBI Villa Clara. Además, lo fue la Revista Ciencias de la Información, de carácter científico.

Atendiendo al análisis formal se identifica que el logotipo está formado por las siglas ASCUBI, de color negro, seguido de la palabra Villa Clara del mismo color. La selección y disposición de la tipografía, incluida la fuente, constituyen elementos positivos que favorecen la legibilidad del enunciado. La impresión en blanco y negro favorece de manera forzada la unidad visual en cuanto a los elementos analizados. Desde el comportamiento en blanco y negro se observa que la impresión del documento en blanco y negro va en detrimento de la utilización de la información los códigos de colores Red, Green, Blue (RGB) que le hubiera aportado mayor visualidad al boletín y complementado con los 
colores corporativos de la ASCUBI. La foto empleada del conjunto escultórico se constata de forma quemada. Esto remite al proceso de impresión de la técnica de impresión de los años 90 , periodo en que circula el boletín.

Desde la pregnancia-síntesis se establece que la sencillez tipográfica resultó el elemento distintivo del boletín. Esto evidencia una sincronización con la tendencia mundial en cuanto al diseño de los signos identificadores de las publicaciones seriadas. Al diseño se le señala que no constituye un buen referente desde el punto de vista formal. En sentido general se puede declarar que no es una forma pregnante por su irrelevancia y ausencia de valores en cuanto al diseño.

\section{Conclusiones}

El boletín ASCUBI Villa Clara distinguió a la Filial villaclareña de las del resto de Cuba. Su papel en la divulgación del acontecer y resultados de la profesión en el territorio, así como, en el reconocimiento de la labor desarrollada por insignes figuras del gremio. El surgimiento del boletín potenció que se desarrollaran otras publicaciones que existieron en la filial de forma efímera como el Boletín Exlibri, Boletín Informativo y Reportes de Información.

En la publicación prima la presencia de endogamia editorial identificada en las firmas de las contribuciones. Hay mayor presencia de las temáticas de bibliografía, ASCUBI, comité, actividades y noticias, de acuerdo con la finalidad de la publicación y la propiedad intelectual de la publicación. Las secciones del boletín no variaron de un número a otro y se presentaron de forma sistemática durante su existencia: directiva de la asociación, bibliografía, cursos y noticias.

El boletín se caracterizó por una sencillez topográfica y de diseño. Se emplearon elementos de identidad acorde a la provincia, en detrimento a otros de la profesión. En la publicación se observan las tendencias de diseño de la década de 1990 predominantes en Cuba. El boletín durante el período que circuló fue el resultado del esfuerzo de los miembros del Comité Editorial ante los escasos recursos, aspecto que se refleja en el diseño editorial. 


\section{Referencias bibliográficas}

Aguiar, M. (1990). Nota de la Redacción del boletín ASCUBI Villa Clara 1990. En: Boletín ASCUBI Villa Clara, 1(1): 1-2.

Aguiar, M. (1991). De nuestra portada: Conjunto Escultórico Comandante Ernesto Che Guevara. En: Boletín ASCUBI Villa Clara, 1-2(1-2): 2-4.

Aguiar, M. (2016). Entrevista a Miriam Aguiar. Comunicación personal. Santa Clara, Cuba. Alomá, C. (2016). Entrevista a Cristina Alomá. Comunicación personal. Santa Clara, Cuba. Aparicio, L.A. (2012). La Asociación Cubana de Bibliotecarios (ASCUBI) en Villa Clara (1987-2007). Trabajo de diploma presentado para optar por el título de Licenciado en Ciencias de la Información. Universidad Central "Marta Abreu" de Las Villas.

Bellas, M. (2006). La Asociación Cubana de Bibliotecarios. En: Bibliotecas. Anales de Investigación, 2(2006): 1-16.

Bellas, M. (2007). La Asociación Cubana de Bibliotecarios, un enfoque desde la teoría de las organizaciones. En: Bibliotecas. Anales de Investigación, 3(2007): 1-20.

Bellas-Vilariño, M. (2008). Nota editorial de Margarita Bellas. En: Boletín ASCUBI informa, 2(6): 2-3.

Bellas, M. (2011). La Asociación Cubana de Bibliotecarios: examen histórico de su papel en el desarrollo del campo bibliotecológico nacional. Tesis presentada para optar por el título de máster en Bibliotecología y Ciencias de la Información. Universidad de La Habana.

Boletín ASCUBI Villa Clara. (1992). Nota de la Redacción del boletín ASCUBI Villa Clara 1991-1992. En: Boletín ASCUBI Villa Clara. 1-2(1-2): 2-3.

Cárdenas, M.R., Estévez, V. y Hernández, M. (2011). Diseño del Boletín Informativo para la filial ASCUBI-Villa Clara. Ponencia presentada en la XI Jornada CientíficoBibliotecológica Provincial de Villa Clara. Santa Clara: Feijóo.

Frías, M. (2008). La enseñanza bibliotecológica en Cuba: orígenes y factores condicionantes. En: Acimed, 17(4): 1-10.

Hernández, R., Fernández, C. y Baptista, P. (2006). Metodología de la Investigación. Ciudad de México: Mc Graw Hill.

Hernández, E. A. y Paz, L. E. (2016). La publicidad de bien público y la educación sexual en Cuba. En: Grafica, 4(8): 105-115. 
Hernández, Y. (2002). Las Asociaciones Bibliotecarias Cubanas (1938-1959). Trabajo de diploma presentado para optar por el título de Licenciado en Ciencias de la Información. Universidad de La Habana.

Montes de Oca, D. y Rivera, Z. (2006). María Teresa Freyre de Andrade: fundadora de la bibliotecología cubana. En: Acimed, 14(3): 1-25.

Paz, L.E. (2015). "Reportes de Información", oferta de información Web para la Filial ASCUBI-Villa Clara. Trabajo de diploma presentado para optar por el título de Licenciado en Ciencias de la Información. Universidad Central "Marta Abreu" de Las Villas.

Paz, L. E., Hernández, E. A., Tamayo, D., y Frías, M. (2016). El diseño de ofertas de información basado en la experiencia de usuarios. El caso del boletín Reportes de Información. En: Métodos de información, 7(12): 89-116.

Pedroso, E. (2005). Peculiaridades del desarrollo de las ciencias de la información en Cuba. En: Acimed, 7(47): 1-59.

Pérez, N. E. (2005). La formación de bibliotecaria en Cuba: una mirada a través de los documentos. En: Acimed, 13(3): 1-15.

Pérez, N. E. (2007). La literatura bibliológica-informativa en Cuba. Parte I. Reflexión histórica. En: Acimed, 15(1): 1-17.

Rivera, Z. (2005). Lyceum Lawn Tennis Club: El inicio de la formación de los bibliotecarios en Cuba. En: Bibliotecas. Anales de Investigación, 1(2005): 80-95.

Rivera, Z. (2008). Bibliotecología cubana de la República: examen histórico de su desarrollo. Tesis presentada para optar por el título de Doctor en Documentación e Información Científica. Universidad de la Habana y Universidad de Granada.

Viciedo, M. (2005). Biblioteca Pública y Revolución. Su desarrollo de 1959-1989. Tesis presentada para optar por el título de máster en Bibliotecología y Ciencias de la Información. Universidad de La Habana.

Vivero, N., Rivera, Z., Linares, R. y Botana, M. (2007). La revista Cuba Bibliotecológica: reflejo del desarrollo de la bibliotecología cubana en la década de los años 1950. En: Acimed, 16(6): 1-54. 


\section{Nota de los Autores}

Maylin Frías Guzmán: Doctora en Documentación e Información Científica. Departamento de Ciencias de la Información. Universidad Central "Marta Abreu" de Las Villas, Cuba. EMail: maylinfguz@gmal.com

Luis Ernesto Paz Enrique: Licenciado en Ciencias de la Información. Dirección de Información y Documentación Científica. Universidad Central "Marta Abreu" de Las Villas, Cuba. E-mail: luisernestope@uclv.cu

Cristina Alomá Cires: Máster en Educación Superior. Biblioteca Provincial "Marti" de Villa Clara, Cuba.E-mail: bpmarti@cult.vcl.cu

Deymis Tamayo Rueda: Licenciada en Bibliotecología y Ciencias de la Información. Departamento de Ciencias de la Información. Universidad Central "Marta Abreu" de Las Villas, Cuba. E-mail: deymist@uclv.cu

Eduardo Alejandro Hernández Alfonso: Licenciado en Comunicación Social. Departamento de Extensión Universitaria, Universidad Central "Marta Abreu" de Las Villas, Cuba. E-mail: ealejandro@uclv.cu 